\title{
BALLS LEFT EMPTY BY A CRITICAL BRANCHING WIENER PROCESS
}

\author{
PÁL RÉVÉSZ \\ Technische Universität Wien \\ Institut für Statistik und Wahrscheinlichkeitstheorie \\ A-1040 Wien, Austria
}

(Received July, 1996; Revised October, 1996)

\begin{abstract}
At time $t=0$ we have a Poisson random field on $\mathbb{R}^{d}$. Each particle executes a critical branching Wiener process starting from its position at time $t=0$. Let $R_{T}$ be the radius of the largest ball around the origin of $\mathbb{R}^{d}$ which does not contain any particle at time $T$. Our goal is to characterize the properties of the stochastic process $\left\{R_{T}, T \geq 0\right\}$.
\end{abstract}

This article is dedicated to the memory of Professor Roland L. Dobrushin.

Key words: Branching Random Process, Branching Random Fields, Strong Laws.

AMS (MOS) subject classifications: $60 \mathrm{~J} 80,60 \mathrm{~K} 35$.

\section{Introduction}

Consider the following

Model 1:

(i) a particle starts from the position $0 \in \mathbb{R}^{d}$ and executes a Wiener process $W(t) \in \mathbb{R}^{d}$;

(ii) arriving at time $t=1$ to the new location $W(1)$, it dies;

(iii) at death, it is replaced by $Y$ offspring, where

$$
\mathbf{P}\{Y=0\}=\mathbf{P}\{Y=2\}=1 / 2 ;
$$

(iv) each offspring, starting from where its ancestor dies, executes a Wiener process (from its starting point) and repeats the above given steps and so on. All Wiener processes and offspring numbers are assumed independent of each other.

A more formal definition is given in Chapter 6 of [1], p. 91.

Let $A \subset \mathbb{R}^{d}$ be a Borel set and let $\lambda(A, t)(t=0,1,2, \ldots)$ be the number of particles located in $A$ at time $t$. Then

$$
B(t)=\lambda\left(\mathbb{R}^{d}, t\right)
$$

is the number of particles living at $t$ and $\{B(t), t=0,1,2, \ldots\}$ is a branching process.

We also consider the following

Model 2: At time $t=0$ we have a Poisson random field of parameter $\mu$, i.e., in a Borel set $A \subset \mathbb{R}^{d}$, we have $k$ particles with probability 


$$
\pi(A, k)=\frac{(\mu|A|)^{k}}{k !} e^{-\mu|A|}
$$

where $|A|$ is the Lebesgue measure of $A$. It is also assumed that the numbers of particles in disjoint Borel sets are independent r.v.'s. Each particle executes a critical branching Wiener process (starting from its position at time $t=0$ ) according to Model 1.

A more formal definition is given in Chapter 8 of [1], p. 129. Let $\Lambda(A, t)$ be the number of particles located in $A$ at time $t$. Then clearly

$$
\mathbf{P}\{\Lambda(A, 0)=k\}=\pi(A, k) .
$$

Let

and

$$
\mathcal{C}(x, r)=\{y:\|y-x\| \leq r\} \subset \mathbb{R}^{d}
$$

$$
R_{T}=\sup \{R: \Lambda(\mathcal{C}(0, R), T)=0\} \quad(T=0,1,2, \ldots)
$$

i.e., $R_{T}$ is the radius of the largest ball around the origin of $\mathbb{R}^{d}$ which does not contain any particle at time $T$.

We are interested in the limit behavior of $R_{T}$ as $T \rightarrow \infty$.

In the case $d=1$, this problem is very simple. In fact we have,

Theorem A: (Theorem 8.2 p. 129 in [1]). Let $d=1$. Then for any $\epsilon>0$ we have

$$
\Lambda\left(\mathcal{C}\left(0, T(\log T)^{-1-\epsilon}\right), T\right)=0 \text { a.s. }
$$

for all but finitely many $T$,

and

$$
\begin{gathered}
\Lambda(\mathcal{C}(0, \epsilon T), T) \geq 1 \text { i.o. a.s., } \\
\Lambda\left(\mathcal{C}\left(0, \epsilon^{-1} T\right), T\right)=0 \text { i.o. a.s., }
\end{gathered}
$$

$$
\Lambda\left(\mathcal{C}\left(0, T(\log T)^{1+\epsilon}\right), T\right) \geq 1 \text { a.s. }
$$

for all but finitely many $T$.

We note that Theorem 8.2 of [1] is formulated in a slightly different way, but the above Theorem A can be obtained directly by the method presented there.

Now we formulate our main result.

Theorem 1: We have

for all but finitely many $T$,

$$
\Lambda\left(\mathcal{C}\left(0, R_{1}(T, d)\right), T\right) \geq 1 \text { a.s. }
$$

$$
\begin{gathered}
\Lambda\left(\mathcal{C}\left(0, R_{2}(T, d)\right), T\right)=0 \text { i.o. a.s., } \\
\Lambda\left(\mathcal{C}\left(0, R_{3}(T, d)\right), T\right) \geq 1 \quad \text { i.o. } \quad \text { a.s. }
\end{gathered}
$$

and

$$
\Lambda\left(\mathcal{C}\left(0, R_{4}(T, d)\right), T\right)=0 \text { a.s. }
$$

for all but finitely many $T$, where

$$
R_{1}(T, d)=\left\{\begin{array}{cl}
T(\log T)^{1+\epsilon} & \text { if } d=1, \\
K(T \log T)^{1 / 2} & \text { if } d=2, \\
K(\log T)^{1 /(d-2)} & \text { if } d \geq 3
\end{array}\right.
$$




$$
\begin{aligned}
& R_{2}(T, d)=\left\{\begin{array}{cc}
\epsilon^{-1} T & \text { if } d=1, \\
T^{1 / 2}(g(T))^{-1} & \text { if } d=2, \\
K^{-1}(\log \log \log T)^{1 /(d-2)} & \text { if } d \geq 3,
\end{array}\right. \\
& R_{3}(T, d)=\left\{\begin{array}{cc}
\epsilon T & \text { if } d=1, \\
(\log T)^{-1 / 2+\epsilon} & \text { if } d=2, \\
K^{-1}(\log T)^{-1 / d} & \text { if } d \geq 3, \\
T(\log T)^{-1-\epsilon} & \text { if } d=1, \\
T^{-1 / 2}(\log T)^{-1 / 2-\epsilon} & \text { if } d=2, \\
T^{-1 / d}(\log T)^{-1 / d-\epsilon} & \text { if } d \geq 3,
\end{array}\right.
\end{aligned}
$$

$K$ is large enough, $g(T)$ is an arbitrary function with $g(T) \uparrow \infty$ and $\epsilon$ is an arbitrary positive number.

Remark: Intuitively it is clear that if $R_{1}(T)(T=1,2, \ldots)$ is a function going to infinity fast enough, then the ball around the origin, of radius $R_{1}(T)$ will contain at least one living particle at time $T$ for any $T$ large enough. Theorem 1 claims that in $\mathbb{R}^{2}$ we might choose $R_{1}(T)=$ $K(T \log T)^{1 / 2}$, while in $\mathbb{R}^{3}$ it is enough to choose $R_{1}(T)=K \log T$. We are also interested to characterize those functions $R_{3}(T)$ for which it is still true that the ball, around the origin, of radius $R_{3}(T)$ contains particles at time $T$ for infinitely many $T$. Theorem 1 claims that in $\mathbb{R}^{2}$ we might choose $R_{3}(T)=(\log T)^{-1 / 2+\epsilon}$ while in $\mathbb{R}^{3}$ we might have $R_{3}(T)=K^{-1}(\log T)^{-1 / 3}$. The results on $R_{2}$ and $R_{4}$ tell us how exact are the results on $R_{1}$ and $R_{3}$. Unfortunately, it turns out that we have a very big gap.

We also prove two theorems describing some properties of $\lambda(\cdot, \cdot)$ of Model 1 , which will be used in the proof of Theorem 1 and which seem to be interesting in themselves. and let

Let $f(t) \quad(t=1,2, \ldots)$ be a positive, real valued function with $f(t) \rightarrow \infty$ (as $t \rightarrow \infty)$, let $\alpha \in \mathbb{R}^{d}$

$$
\mathcal{C}=\mathcal{C}\left(\alpha T^{1 / 2}, T^{1 / 2}(f(T))^{-1}\right)
$$

Then we have,

Theorem 2: In case $d=1$ we have

$$
\begin{gathered}
1+(1-\epsilon)\left(1-\frac{1}{K}\right) \frac{c(\alpha)}{2} \frac{T}{f(T)} \leq \mathbf{E}(\lambda(\mathcal{C}, T) \mid \lambda(\mathcal{C}, T)>0) \\
\leq 1+(1+\epsilon) c(\alpha) \frac{T}{f(T)}+K \frac{T}{f^{2}(T)}
\end{gathered}
$$

for any $K>0, \epsilon>0$ if $T$ is large enough, where

$$
c(\alpha)=\left(\frac{2}{\pi}\right)^{1 / 2} \int_{0}^{\pi / 2} \exp \left(-\frac{\alpha^{2}}{2} \frac{1-\sin x}{1+\sin x}\right) d x .
$$

If we also assume that $f(T) \leq T^{1 / 2}$ and $K \geq 2$ then

$$
\begin{gathered}
1+(1-\epsilon)\left(1-\frac{1}{K}\right) \frac{c(\alpha)}{2} \frac{T}{f(T)} \\
\leq \mathbf{E}(\lambda(\mathcal{C}, T) \mid \lambda(\mathcal{C}, T)>0) \leq 1+(1+\epsilon) \frac{c(\alpha)}{2}\left(1+\frac{2}{K}\right) \frac{T}{f(T)}+K \frac{T}{f^{2}(T)} .
\end{gathered}
$$


In case $d=2$ we have

$$
\begin{aligned}
& 1+(1-\epsilon)\left(1-\frac{1}{K}\right) \frac{1}{4} \frac{T}{f^{2}(T)} \log f(T) \\
& \leq \mathbf{E}(\lambda(\mathcal{C}, T) \mid \lambda(\mathcal{C}, T)>0) \leq 1+\frac{1}{2}(1+\epsilon) \frac{T}{f^{2}(T)} \log f(T)+K \frac{T}{f^{2}(T)}
\end{aligned}
$$

for any $K>0$ if $T$ is large enough.

If we also assume that $f(T) \leq T^{1 / 2}$ and $K \geq 2$, then

$$
\begin{gathered}
1+(1-\epsilon)\left(1-\frac{1}{K}\right) \frac{1}{4} \frac{T}{f^{2}(T)} \log f(T) \\
\leq \mathbf{E}(\lambda(\mathrm{e}, T) \mid \lambda(\mathrm{e}, T)>0) \\
\leq 1+\frac{1+\epsilon}{4}\left(1+\frac{2}{K}\right) \frac{T}{f^{2}(T)} \log f(T)+K \frac{T}{f^{2}(T)} .
\end{gathered}
$$

In case $d \geq 3$ for any $K>0$ if $T$ is large enough, we have

$$
\begin{gathered}
1+(1-\epsilon)\left(1-\frac{1}{K}\right) \frac{1}{d-2} \frac{\omega_{d}}{(4 \pi)^{d / 2}} \frac{T}{f^{2}(T)} K^{-(d-2) / 2} \\
\leq E(\lambda(\mathcal{C}, T) \mid \lambda(\mathcal{C}, T)>0) \\
\leq 1+(1+\epsilon) \frac{2}{d-2} \frac{\omega_{d}}{(4 \pi)^{d / 2}} \frac{T}{f^{2}(T)} K^{-(d-2) / 2}+K \frac{T}{f^{2}(T)},
\end{gathered}
$$

where

$$
\omega_{d}=\left\{\begin{array}{cc}
2 & \text { if } d=1, \\
\pi & \text { if } d=2, \\
\frac{\pi^{d / 2}}{\Gamma(d / 2+1)} & \text { if } d \geq 3
\end{array}\right.
$$

is the volume of a ball in $\mathbb{R}^{d}$ of radius 1 .

Consequences: In case $d=1$,

$$
\begin{aligned}
& \frac{c(\alpha)}{2} \leq \liminf _{T \rightarrow \infty} \frac{f(T)}{T} \mathbf{E}(\lambda(\mathcal{C}, T) \mid \lambda(\mathcal{C}, T)>0) \\
\leq & \limsup _{T \rightarrow \infty} \frac{f(T)}{T} \mathbf{E}(\lambda(\mathcal{C}, T) \mid \lambda(\mathcal{C}, T)>0)<c(\alpha),
\end{aligned}
$$

provided that

$$
\text { If } d=1 \text { and }
$$

$$
\lim _{T \rightarrow \infty} \frac{T}{f(T)}=\infty
$$

then

$$
\lim _{T \rightarrow \infty} \frac{T}{f(T)}=\beta, \quad 0 \leq \beta<\infty
$$

$$
\begin{aligned}
& 1+\frac{c(\alpha)}{2} \beta \leq \liminf _{T \rightarrow \infty} \mathbf{E}(\lambda(\mathcal{C}, T) \mid \lambda(\mathcal{C}, T)>0) \\
\leq & \limsup _{T \rightarrow \infty} \mathbf{E}(\lambda(\mathcal{C}, T) \mid \lambda(\mathcal{C}, T)>0) \leq 1+c(\alpha) \beta .
\end{aligned}
$$

If $d=1$ and $f(T) \leq T^{1 / 2}$, then 


$$
\lim _{T \rightarrow \infty} \frac{f(T)}{T} \mathbf{E}(\lambda(\mathcal{C}, T) \mid \lambda(\mathcal{C}, T)>0)=\frac{c(\alpha)}{2}
$$

Note that

In case $d=2$ we have

$$
c(0)=\left(\frac{\pi}{2}\right)^{1 / 2}
$$

$$
\begin{aligned}
& \quad \frac{1}{4} \leq \liminf _{T \rightarrow \infty} \frac{f^{2}(T)}{T \log f(T)} \mathbf{E}(\lambda(\mathcal{C}, T) \mid \lambda(\mathcal{C}, T)>0) \\
& \leq \limsup _{T \rightarrow \infty} \frac{f^{2}(T)}{T \log f(T)} \mathbf{E}(\lambda(\mathcal{C}, T) \mid \lambda(\mathcal{C}, T)>0) \leq \frac{1}{2}
\end{aligned}
$$

provided that

If $d=2$ and

$$
\lim _{T \rightarrow \infty} \frac{T}{f^{2}(T)} \log f(T)=\infty
$$

then

$$
\lim _{T \rightarrow \infty} \frac{T}{f^{2}(T)} \log f(T)=\beta
$$

$$
\begin{aligned}
& 1+\frac{\beta}{4} \leq \liminf _{T \rightarrow \infty} \mathbf{E}(\lambda(\mathcal{C}, T) \mid \lambda(\mathcal{C}, T)>0) \\
\leq & \limsup _{T \rightarrow \infty} \mathbf{E}(\lambda(\mathcal{C}, T) \mid \lambda(\mathcal{C}, T)>0) \leq 1+\frac{\beta}{2} .
\end{aligned}
$$

If $d=2$ and $f(T) \leq T^{1 / 2}$, then

$$
\lim _{T \rightarrow \infty} \frac{f^{2}(T)}{T \log f(T)} \mathbf{E}(\lambda(\mathcal{C}, T) \mid \lambda(\mathcal{C}, T)>0)=\frac{1}{4} .
$$

In case $d \geq 3$,

$$
\begin{gathered}
\quad \frac{2}{d-2} \frac{\omega_{d}}{(8 \pi)^{d / 2}} \leq \liminf _{T \rightarrow \infty} \frac{f^{2}(T)}{T} \mathbf{E}(\lambda(\mathcal{C}, T) \mid \lambda(\mathcal{C}, T)>0) \\
\leq \limsup _{T \rightarrow \infty} \frac{f^{2}(T)}{T} \mathbf{E}(\lambda(\mathcal{C}, T) \mid \lambda(\mathcal{C}, T)>0) \leq \frac{4}{d-2} \frac{\omega_{d}}{(8 \pi)^{d / 2}}+2,
\end{gathered}
$$

provided that

$$
\lim _{T \rightarrow \infty} \frac{T}{f^{2}(T)}=\infty
$$

If $d \geq 3$ and

$$
\lim _{T \rightarrow \infty} \frac{T}{f^{2}(T)}=\beta
$$

then

$$
\begin{gathered}
1+\frac{2}{d-2} \frac{\omega_{d}}{(8 \pi)^{d / 2}} \beta \leq \liminf _{T \rightarrow \infty} \mathbf{E}(\lambda(\mathcal{C}, T) \mid \lambda(\mathcal{C}, T)>0) \\
\leq \limsup _{T \rightarrow \infty} \mathbf{E}(\lambda(\mathcal{C}, T) \mid \lambda(\mathcal{C}, T)>0) \\
\leq 1+2 \beta+\frac{4}{d-2} \frac{\omega_{d}}{(8 \pi)^{d / 2}} \beta
\end{gathered}
$$

Theorem 3: Consider the case where

Then,

$$
d=1, \lim _{T \rightarrow \infty} \frac{T}{f(T)}=\infty .
$$

$$
\frac{1}{(2 \pi)^{1 / 2}} \frac{\exp \left(-\alpha^{2} / 2\right)}{c(\alpha)} \leq \liminf _{T \rightarrow \infty}\{\lambda(e, T)>0 \mid B(T)>0\}
$$




$$
\begin{gathered}
\leq \limsup _{T \rightarrow \infty} \mathbf{P}\{\lambda(\mathcal{C}, T)>0 \mid B(T)>0\} \\
\leq\left(\frac{2}{\pi}\right)^{1 / 2} \frac{\exp \left(-\alpha^{2} / 2\right)}{c(\alpha)}
\end{gathered}
$$

If $d=1$ and

then

$$
\lim _{T \rightarrow \infty} \frac{T}{f(T)}=\beta, \quad 0 \leq \beta<\infty
$$

If

$$
\begin{gathered}
\frac{\beta}{(2 \pi)^{1 / 2}} \frac{\exp \left(-\alpha^{2} / 2\right)}{1 \leq c(\alpha) \beta} \leq \liminf _{T \rightarrow \infty} \frac{f(T)}{T} \mathbf{P}\{\lambda(\mathcal{C}, T)>0 \mid B(T)>0\} \\
\leq \limsup _{T \rightarrow \infty} \frac{f(T)}{T} \mathbf{P}\{\lambda(\mathcal{C}, T)>0 \mid B(T)>0\} \\
\leq \frac{\beta}{(2 \pi)^{1 / 2}} \frac{2 \exp \left(-\alpha^{2} / 2\right)}{2+c(\alpha) \beta}
\end{gathered}
$$

then

$$
d=1 \text { and } f(T) \leq T^{1 / 2} \text {, }
$$

$$
\lim _{T \rightarrow \infty} \mathbf{P}\{\lambda(\mathcal{C}, T)>0 \mid B(T)>0\}=\left(\frac{2}{\pi}\right)^{1 / 2} \frac{e^{-\alpha^{2} / 2}}{c(\alpha)} .
$$

Now consider the case

Then,

$$
d=2, \lim _{T \rightarrow \infty} \frac{T}{f^{2}(T)} \log f(T)=\infty
$$

$$
\begin{gathered}
\frac{1}{2} e^{-\alpha^{2} / 2} \leq \liminf _{T \rightarrow \infty}(\log f(T)) \mathbf{P}\{\lambda(\mathcal{C}, T)>0 \mid B(T)>0\} \\
\leq \limsup _{T \rightarrow \infty}(\log f(T)) \mathbf{P}\{\lambda(\mathcal{C}, T)>0 \mid B(T)>0\} \\
\leq e^{-\alpha^{2} / 2} .
\end{gathered}
$$

If

then

$$
d=2 \quad \text { and } \lim _{T \rightarrow \infty} \frac{T}{f^{2}(T)} \log f(T)=\beta,
$$

$$
\begin{gathered}
\frac{\exp \left(-\alpha^{2} / 2\right)}{4+2 \beta} \leq \liminf _{T \rightarrow \infty} \frac{f^{2}(T)}{T} \mathbf{P}\{\lambda(\mathcal{C}, T)>0 \mid B(T)>0\} \\
\leq \limsup _{T \rightarrow \infty} \frac{f^{2}(T)}{T} \mathbf{P}\{\lambda(\mathcal{C}, T)>0 \mid B(T)>0\} \\
\leq \frac{\exp \left(-\alpha^{2} / 2\right)}{4+\beta}
\end{gathered}
$$

If

then

$$
d=2, \lim _{T \rightarrow \infty} \frac{T}{f^{2}(T)} \log f(T)=\infty \text { and } f(T) \leq T^{1 / 2},
$$

$$
\lim _{T \rightarrow \infty}(\log f(T)) \mathbf{P}\left\{\lambda(\mathcal{C}, T)>0|B(T)>0|=\exp \left(-\frac{\alpha^{2}}{2}\right)\right. \text {. }
$$

Now consider the case

Then,

$$
d \geq 3, \quad \lim _{T \rightarrow \infty} \frac{T}{f^{2}(T)}=\infty .
$$

$$
\frac{2^{d} \omega_{d}(d-2) e^{-\alpha^{2} / 2}}{8 \omega_{d}+4(8 \pi)^{d / 2}(d-2)}
$$




$$
\begin{aligned}
& \leq \liminf _{T \rightarrow \infty}(f(T))^{d-2} \mathbf{P}\{\lambda(\mathcal{C}, T)>0 \mid B(T)>0\} \\
& \leq \limsup _{T \rightarrow \infty}(f(T))^{d-2} \mathbf{P}\{\lambda(\mathcal{C}, T)>0 \mid B(T)>0\} \\
& \quad \leq(d-2) 2^{d-2} \exp \left(-\frac{\alpha^{2}}{2}\right)
\end{aligned}
$$

If

then

$$
\begin{gathered}
d \geq 3 \text { and } \lim _{T \rightarrow \infty} \frac{T}{f^{2}(T)}=\beta, \\
\frac{2^{d} \omega_{d}(d-2) e^{-\alpha^{2} / 2}}{8 \beta \omega_{d}+2(d-2)(8 \pi)^{d / 2}(1+\beta)} \\
\leq \liminf _{T \rightarrow \infty} \frac{(f(T))^{d}}{T} \mathbf{P}\{\lambda(\mathcal{C}, T)>0 \mid B(T)>0\} \\
\leq \limsup _{T \rightarrow \infty} \frac{(f(T))^{d}}{T} \mathbf{P}\{\lambda(\mathcal{C}, T)>0 \mid B(T)>0\} \\
\leq \frac{(d-2) 2^{d} \omega_{d} e^{-\alpha^{2} / 2}}{2(d-2)(8 \pi)^{d / 2}+4 \omega_{d} \beta} .
\end{gathered}
$$

\section{Lemmas}

Let

$$
\begin{aligned}
& W_{1}(t)=\left\{W_{11}(t), W_{12}(t), \ldots, W_{1 d}(t)\right\} \\
& W_{2}(t)=\left\{W_{21}(t), W_{22}(t), \ldots, W_{2 d}(t)\right\} \\
& W_{3}(t)=\left\{W_{31}(t), W_{32}(t), \ldots, W_{3 d}(t)\right\}
\end{aligned}
$$

be independent Wiener processes and let

$$
\begin{aligned}
& \Gamma_{1}(t, s, T)=\left\{\begin{array}{cc}
W_{1}(t) & \text { if } 0 \leq t \leq s, \\
W_{1}(s)+W_{2}(t-s) & \text { if } s \leq t \leq T,
\end{array}\right. \\
& \Gamma_{2}(t, s, T)=\left\{\begin{array}{cc}
W_{1}(t) & \text { if } 0 \leq t \leq s, \\
W_{1}(s)+W_{3}(t-s) & \text { if } s \leq t \leq T .
\end{array}\right.
\end{aligned}
$$

Let

$$
\gamma(x)=\gamma_{T, s}(x)=\mathbf{P}\left\{\Gamma_{2}(T, s, T)=x \mid \Gamma_{1}(T, s, T)=z\right\}
$$

be the conditional density function of $\Gamma_{2}$ given $\Gamma_{1}=z$.

Lemma 1:

where

$$
\begin{aligned}
& \gamma(x)=\left(2 \pi \sigma^{2}\right)^{-d / 2} \exp \left(-\frac{(x-\nu)^{2}}{2 \sigma^{2}}\right), \\
& \nu=\mathbf{E}\left(\Gamma_{2}(T, s, T) \mid \Gamma_{1}(T, s, T)=z\right)=\frac{s z}{T}
\end{aligned}
$$

and

$$
\sigma^{2}=\mathbf{E}\left(\left(\Gamma_{2}(T, s, T)-\nu\right)^{2} \mid \Gamma_{1}(T, s, T)=z\right)=T\left(1-\frac{s^{2}}{T^{2}}\right) .
$$

Proof is trivial.

Lemma 2: Let $A \subseteq \mathbb{R}^{d}$ be a Borel set. Then 
where

$$
\mathbf{P}\left\{\Gamma_{2}(T, s, T) \in A \mid \Gamma_{1}(T, s, T) \in A\right\}=\frac{\int_{A}\left(\int_{A} \gamma(x) d x\right) \psi(z) d z}{\int_{A} \psi(z) d z},
$$

is the density function of $\Gamma_{1}(T, s, T)$.

$$
\psi(z)=\psi_{T}(z)=(2 \pi T)^{-d / 2} \exp \left(-\frac{z^{2}}{2 T}\right)
$$

Proof: Since

Lemma 2 follows.

$$
\mathbf{P}\left\{\Gamma_{2} \in A \mid \Gamma_{1} \in A\right\}=\frac{\int_{A} \mathbf{P}\left\{\Gamma_{2} \in A \mid \Gamma_{1}=z\right\} \psi(z) d z}{\int_{A} \psi(z) d z},
$$

Lemma 3: Let

and

$$
\kappa_{T}=T-K \frac{T}{f^{2}(T)} \quad(K>0)
$$

$$
P(T)=\sum_{s=1}^{\kappa} T\left\{\Gamma_{2} \in \mathrm{C} \mid \Gamma_{1} \in \mathrm{C}\right\} .
$$

Then in the case $d=1$, for any $\epsilon>0$, there exists a $T_{0}=T_{0}(\epsilon)>0$ such that

$$
(1-\epsilon)\left(1-\frac{1}{K}\right) c(\alpha) \leq \frac{f(T)}{T} P(T) \leq(1+\epsilon) c(\alpha)
$$

if $T \geq T_{0}$, where

$$
c(\alpha)=\left(\frac{2}{\pi}\right)^{1 / 2} \int_{0}^{\pi / 2} \exp \left(-\frac{\alpha^{2}}{2} \frac{1-\sin x}{1+\sin x}\right) d x .
$$

In the case $d=2$, for any $\epsilon>0$, there exists a $T_{0}=T_{0}(\epsilon)>0$ such that

$$
(1-\epsilon)\left(1-\frac{1}{K}\right) \frac{1}{2} \frac{T}{f^{2}(T)} \log f(T) \leq P(T) \leq(1+\epsilon) \frac{1}{2} \frac{T}{f^{2}(T)} \log f(T)
$$

if $T \geq T_{0}$.

In the case $d \geq 3$, for any $\epsilon>0$, there exists a $T_{0}=T_{0}(\epsilon)>0$ such that

$$
\begin{aligned}
& (1-\epsilon)\left(1-\frac{1}{K}\right) \frac{\omega_{d}}{(4 \pi)^{d / 2}} \frac{2}{d-2} \frac{T}{f^{2}(T)} K^{-(d-2) / 2} \\
& \leq P(T) \leq(1+\epsilon) \frac{\omega_{d}}{(4 \pi)^{d / 2}} \frac{2}{d-2} \frac{T}{f^{2}(T)} K^{-(d-2) / 2}
\end{aligned}
$$

if $T \geq T_{0}$.

Proof: By Lemma 2,

$$
P(T)=\frac{\sum_{s=1}^{\kappa_{T}} \iint_{\mathrm{e}} \int_{\mathrm{e}} \gamma(x) d x \psi(z) d z}{\int_{\mathrm{C}} \psi(z) d z} .
$$

Let

$$
\begin{aligned}
& x=\alpha T^{1 / 2}+u \frac{T^{1 / 2}}{f(T)} \\
& z=\alpha T^{1 / 2}+v \frac{T^{1 / 2}}{f(T)} .
\end{aligned}
$$

Then,

$$
u \in \mathfrak{C}(0,1) \text { and } v \in \mathbb{C}(0,1)
$$


if

Hence,

$$
x \in \mathcal{C} \text { and } z \in \mathrm{C} \text {. }
$$

and

$$
\begin{aligned}
\exp \left(-\frac{z^{2}}{2 T}\right) & =\exp \left(-\frac{\alpha^{2}}{2}\right) \exp \left(-\frac{1}{2}\left(\frac{2(\alpha, v)}{f(T)}+\frac{v^{2}}{f^{2}(T)}\right)\right)= \\
& =\exp \left(-\frac{\alpha^{2}}{2}\right)\left(1+O\left(\frac{1}{f(T)}\right)\right) \\
\int_{\mathcal{C}} \psi(z) d z & =(2 \pi T)^{-d / 2}|\mathcal{C}| \exp \left(-\frac{\alpha^{2}}{2}\right)\left(1+O\left(\frac{1}{f(T)}\right)\right)
\end{aligned}
$$

Observe that

$$
P(T)=\left(1+O\left(\frac{1}{f(T)}\right)\right) \frac{1}{|\mathcal{C}|}(2 \pi)^{-d / 2} \sum_{s=1}^{\kappa_{T}} \sigma^{-d} \int_{\mathcal{C}} \int_{\mathcal{C}} \exp \left(-\frac{(x-\nu)^{2}}{2 \sigma^{2}}\right) d x d z
$$

$$
\begin{gathered}
\frac{x-\nu}{\sigma}=\alpha\left(\frac{T-s}{T+s}\right)^{1 / 2}+(u-v) \frac{T}{f(T)((T-s)(T+s))^{1 / 2}}+v\left(\frac{T-s}{T+s}\right)^{1 / 2} \frac{1}{f(T)} \\
\left(\frac{x-\nu}{\sigma^{2}}\right)^{2}=\alpha^{2} \frac{T-s}{T+s}+(u-v)^{2} \frac{T^{2}}{f^{2}(T)\left(T^{2}-s^{2}\right)}+O\left(\frac{1}{f(T)}\right)
\end{gathered}
$$

and

$$
\frac{T}{\sigma^{2} f^{2}(T)}=\frac{T^{2}}{\left(T^{2}-s^{2}\right) f^{2}(T)} \leq \frac{1}{K}
$$

provided that

Since

$$
1 \leq s \leq \kappa_{T}
$$

we have

$$
d x d z=\frac{T^{d}}{(f(T))^{2 d}} d u d v
$$

$$
\begin{gathered}
\int_{\mathcal{C}} \int_{\mathcal{C}} \exp \left(-\frac{(x-\nu)^{2}}{2 \sigma^{2}}\right) d x d z \\
=\left(1+O\left(\frac{1}{f(T)}\right)\right) \frac{T^{d}}{(f(T))^{2 d}} \exp \left(-\frac{\alpha^{2}}{2} \frac{T-s}{T+s}\right) \int_{\mathcal{C}_{(0,1)}} \int_{\mathcal{C}_{(0,1)}} \exp \left(-\frac{(u-v)^{2}}{2} \frac{T}{\sigma^{2} f^{2}(T)}\right) d u d v \\
\leq\left(1+O\left(\frac{1}{f(T)}\right)\right) \frac{T^{d}}{(f(T))^{2 d}} \omega_{d}^{2} \exp \left(-\frac{\alpha^{2}}{2} \frac{T-s}{T+s}\right)
\end{gathered}
$$

and

$$
\int_{\mathcal{C}} \int_{\mathcal{C}} \exp \left(-\frac{(x-\nu)^{2}}{2 \sigma^{2}}\right) d x d z>\left(1+O\left(\frac{1}{f(T)}\right)\right)\left(1-\frac{1}{K}\right) \frac{T^{d}}{(f(T))^{2 d}} \omega_{d}^{2} \exp \left(-\frac{\alpha^{2}}{2} \frac{T-s}{T+s}\right)
$$

Hence

where

$$
\begin{gathered}
\left(1+O\left(\frac{1}{f(T)}\right)\right)\left(1-\frac{1}{K}\right) I \leq P(T) \leq\left(1+O\left(\frac{1}{f(T)}\right)\right) I \\
I=\omega_{d}(2 \pi)^{-d / 2} \frac{T^{d / 2}}{(f(T))^{d}} \sum_{s=1}^{\kappa_{T}} \sigma^{-d} \exp \left(-\frac{\alpha^{2}}{2} \frac{T-s}{T+s}\right)
\end{gathered}
$$




$$
\begin{gathered}
=\omega_{d}(2 \pi)^{-d / 2} \frac{T}{(f(T))^{d}} \sum_{s=1}^{\kappa_{T}} \frac{1}{T}\left(1-\frac{s^{2}}{T^{2}}\right)^{-d / 2} \exp \left(-\frac{\alpha^{2}}{2} \frac{1-s / T}{1+s / T}\right) \\
\sim \omega_{d}(2 \pi)^{-d / 2} \frac{T}{(f(T))^{d}} \int_{0}^{1-K(f(T))^{-2}}\left(1-u^{2}\right)^{-d / 2} \exp \left(-\frac{\alpha^{2}}{2} \frac{1-u}{1+u}\right) d u .
\end{gathered}
$$

In the case $d=1$,

$$
\begin{gathered}
I \sim\left(\frac{2}{\pi}\right)^{1 / 2} \frac{T}{f(T)} \int_{0}^{1}\left(1-u^{2}\right)^{-1 / 2} \exp \left(-\frac{\alpha^{2}}{2} \frac{1-u}{1+u}\right) d u \\
=\left(\frac{2}{\pi}\right)^{1 / 2} \frac{T}{f(T)} \int_{0}^{\pi / 2} \exp \left(-\frac{\alpha^{2}}{2} \frac{1-\sin x}{1+\sin x}\right) d x .
\end{gathered}
$$

Hence, for $d=1$ and for any $K>0$, we have

$$
\left(1+O\left(\frac{1}{f(T)}\right)\right)\left(1-\frac{1}{K}\right) c(\alpha) \frac{T}{f(T)} \leq P(T) \leq\left(1+O\left(\frac{1}{f(T)}\right)\right) c(\alpha) \frac{T}{f(T)}
$$

Hence, Lemma 3 is proved for $d=1$.

In the case $d \geq 2$,

Hence, in the case $d=2$,

$$
\begin{gathered}
I \sim \omega_{d}(2 \pi)^{-d / 2} \frac{T}{(f(T))^{d}} \int_{K(f(T))^{-2}}^{1}(v(2-v))^{-d / 2} \exp \left(-\frac{\alpha^{2}}{2} \frac{v}{2-v}\right) d v \\
\sim \omega_{d}(4 \pi)^{-d / 2} \frac{T}{(f(T))^{d}} \int_{K(f(T))^{-2}}^{1} v^{-d / 2} d v
\end{gathered}
$$

and we have Lemma 3 for $d=2$.

$$
I \sim \frac{1}{2} \frac{T}{f^{2}(T)} \log f(T)
$$

In the case $d \geq 3$,

$$
I \sim \frac{2 \omega_{d}}{d-2}(4 \pi)^{-d / 2} \frac{T}{(f(T))^{2}} \frac{1}{K^{(d-2) / 2}} .
$$

Lemma 4: Let $X, Y$ be i.i.d.r.v.'s with

$$
\begin{gathered}
\mathbf{P}\{X \geq 0\}=\mathbf{P}\{Y \geq 0\}=1, \\
\mathbf{P}\{X>0\}=\mathbf{P}\{Y>0\}=p \quad(0 \leq p \leq 1) . \\
\mathbf{E}(X+Y \mid X+Y>0)=\frac{1}{2-p} \mathbf{E} Y+\mathbf{E}(X \mid X>0) .
\end{gathered}
$$

Then

\section{Proof:}

$$
\begin{gathered}
\mathbf{E}(X+Y \mid X+Y>0)=\frac{2}{\mathbf{P}\{X+Y>0\}} \int_{\{X+Y>0\}} X d \mathbf{P}=\frac{2}{2 p-p^{2}} \mathbf{E} X=\frac{2 p}{2 p-p^{2}} \mathbf{E}(X \mid X>0) \\
=\left(1+\frac{p}{2-p}\right) \mathbf{E}(X \mid X>0)=\mathbf{E}(X \mid X>0)+\frac{1}{2-p} \mathbf{E} Y .
\end{gathered}
$$

Lemma 4 is proved.

Lemma 5:

$$
\mathbf{E}(\lambda(\mathcal{C}, T) \mid B(T)>0) \sim \frac{T}{2}|\mathcal{C}|(2 \pi T)^{-d / 2} \exp \left(-\frac{\alpha^{2}}{2}\right)
$$


Proof: Clearly,

$$
=\frac{T}{2}(2 \pi)^{-d / 2}(f(T))^{-d} \omega_{d} \exp \left(-\frac{\alpha^{2}}{2}\right)
$$

$\mathbf{E}(\lambda(\mathcal{C}, T) \mid B(T))=B(T)(2 \pi T)^{-d / 2} \int_{\mathcal{C}} \exp \left(-\frac{x^{2}}{2 T}\right) d x \sim B(T)|\mathcal{C}|(2 \pi T)^{-d / 2} \exp \left(-\frac{\alpha^{2}}{2}\right)$.

Since

$$
\mathbf{E} B(T)=1 \text { and } \mathbf{P}\{B(T)>0\} \sim \frac{2}{T},
$$

we have

$$
\mathbf{E} \lambda(\mathcal{C}, T)=\mathbf{E E}(\lambda(\mathcal{C}, T) \mid B(T)) \sim(2 \pi T)^{-d / 2}|\mathcal{C}| \exp \left(-\frac{\alpha^{2}}{2}\right)
$$

and

$$
\mathbf{E}(\lambda(\mathcal{C}, T) \mid B(T)>0)=\frac{1}{\mathbf{P}\{B(T)>0\}} \int_{\{B(T)>0\}} \lambda(\mathcal{C}, T) d \mathbf{P}=\frac{1}{\mathbf{P}\{B(T)>0\}} \mathbf{E} \lambda(\mathcal{C}, T)
$$

and consequently we have Lemma 5 .

$$
\text { Lemma 6: } \quad \mathbf{P}\{\lambda(\mathcal{C}, T)>0\} \leq \mathbf{P}\{B(T)>0\} \sim \frac{2}{T} .
$$

Proof is trivial.

\section{Proofs of Theorems 2 and 3}

Having the condition $\{\lambda(\mathrm{C}, T)>0\}$ we have two particles at time $t=1$ and we know that at least one of them has at least one living offspring located in $\mathcal{C}$ at time $T$. Let $\lambda_{11}(\mathcal{C}, T-1)$, respectively $\lambda_{12}(\mathcal{C}, T-1)$ be the number of those offspring of the first respectively, second particle which are located in $C$ at time $T$. Clearly,

$$
\lambda(\mathrm{e}, T)=\lambda_{11}(\mathrm{e}, T-1)+\lambda_{12}(\mathrm{e}, T-1) .
$$

Then by Lemma 4 ,

where

$$
\begin{gathered}
\mathbf{E}(\lambda(\mathcal{C}, T) \mid \Lambda(\mathcal{C}, T)>0) \\
=\mathbf{E}\left(\lambda_{11}(\mathcal{C}, T-1) \mid \lambda_{11}(\mathcal{C}, T-1)>0\right)+\frac{1}{2-p_{1}} \mathbf{E} \lambda_{12}(\mathcal{C}, T-1),
\end{gathered}
$$

$$
p_{1}=\mathbf{P}\left\{\lambda_{11}(\mathrm{C}, T-1)>0\right\}=\mathbf{P}\left\{\lambda_{12}(\mathcal{C}, T-1)>0\right\} .
$$

Consider that particle at time $t=1$ which has at least one offspring living at time $T$ and located in $C$. (In the case both particles have such an offspring, consider one of them.) This particle has at time $t=2$ two offspring and we know that at least one of them has at least one offspring located in $\mathfrak{C}$ at time $T$. Let $\lambda_{21}(\mathfrak{C}, T-2)$ respectively, $\lambda_{22}(\mathfrak{C}, T-2)$ be the number of those offspring of the first respectively, second particle which are located in $C$ at time $T$. Clearly,

Then by Lemma 4 ,

$$
\lambda_{11}(\mathcal{C}, T-1)=\lambda_{21}(\mathcal{C}, T-2)+\lambda_{22}(\mathcal{C}, T-2) .
$$

$$
\begin{gathered}
\mathbf{E}\left(\lambda_{11}(\mathcal{C}, T-1) \mid \lambda_{11}(\mathcal{C}, T-1)>0\right) \\
\mathbf{E}\left(\lambda_{21}(\mathcal{C}, T-2) \mid \lambda_{21}(\mathcal{C}, T-2)>0\right)+\frac{1}{2-p_{2}} \mathbf{E} \lambda_{22}(\mathcal{C}, T-2),
\end{gathered}
$$

where

$$
p_{2}=\mathbf{P}\left\{\lambda_{21}(\mathbb{C}, T-2)>0\right\}=\mathbf{P}\left\{\lambda_{22}(\mathcal{C}, T-2)>0\right\} .
$$

(3.1) and (3.2), combined, imply

$$
\begin{gathered}
\mathbf{E}(\lambda(\mathcal{C}, T) \mid \lambda(\mathcal{C}, T)>0)=\mathbf{E}\left(\lambda_{21}(\mathcal{C}, T-2) \mid \lambda_{21}(\mathcal{C}, T-2)>0\right) \\
+\frac{1}{2-p_{1}} \mathbf{E} \lambda_{12}(\mathcal{C}, T-1)+\frac{1}{2-p_{2}} \mathbf{E} \lambda_{22}(\mathcal{C}, T-2) .
\end{gathered}
$$


Continuing this procedure we obtain

$$
\begin{gathered}
\mathbf{E}(\lambda(\mathrm{C}, T) \mid \lambda(\mathrm{C}, T)>0)=\sum_{s=1}^{T} \frac{1}{2-p_{s}} \mathbf{E} \lambda_{s 2}(\mathrm{C}, T-s) \\
=\sum_{s=1}^{\kappa_{T}} \frac{1}{2-p_{s}} \mathbf{E} \lambda_{s 2}(\mathrm{e}, T-s)+\sum_{s=\kappa_{T}+1}^{T-1} \frac{1}{2-p_{s}} \mathbf{E} \lambda_{s 2}(\mathrm{C}, T-s)+1=I+I I+1
\end{gathered}
$$

where

and

$$
p_{s}=\mathbf{P}\left\{\lambda_{s 1}(\mathrm{C}, T-s)>0\right\}=\mathbf{P}\left\{\lambda_{s 2}(\mathcal{C}, T-s)>0\right\}
$$

Clearly,

$$
\kappa_{T}=T-K \frac{T}{f^{2}(T)}
$$

and

$$
\begin{gathered}
\mathbf{E} \lambda_{s 2}(\mathcal{C}, T-s)=\mathbf{P}\left\{\Gamma_{2}(T, s, T) \in \mathcal{C} \mid \Gamma_{1}(T, s, T) \in \mathcal{C}\right\} \\
0 \leq I I \leq \sum_{s=\kappa_{T}+1}^{T-1} \mathbf{E} \lambda_{s 2}(\mathcal{C}, T-s)=\sum_{s=\kappa_{T}+1}^{T-1} \mathbf{P}\left\{\Gamma_{2} \in \mathcal{C} \mid \Gamma_{1} \in \mathcal{C}\right\} \\
\leq T-\kappa_{T}=K \frac{T}{f^{2}(T)}
\end{gathered}
$$

$$
\left.\frac{1}{2} \sum_{s=1}^{\kappa_{T}} \mathbf{E} \lambda_{s 2}(\mathrm{e}, T-s) \leq I \leq \sum_{s=1}^{\kappa_{T}} \mathbf{E} \lambda_{s 2} \mathrm{e}, T-s\right) .
$$

Then by Lemma $3,(3.4)$ and (3.6) if $d=1$, we have

$$
(1-\epsilon)\left(1-\frac{1}{K}\right) \frac{c(\alpha)}{2} \frac{T}{f(T)} \leq I \leq(1+\epsilon) c(\alpha) \frac{T}{f(T)} .
$$

(3.3), (3.5) and (3.7) imply

for any $K>0$.

$$
\begin{gathered}
1+(1-\epsilon)\left(1-\frac{1}{K}\right) \frac{c(\alpha)}{2} \frac{T}{f(T)} \leq \mathbf{E}(\lambda(\mathcal{C}, T) \mid \lambda(\mathcal{C}, T)>0) \\
\leq 1+(1+\epsilon) c(\alpha) \frac{T}{f(T)}+K \frac{T}{f^{2}(T)}
\end{gathered}
$$

Note that if

then by Lemma 6 ,

$$
f(T) \leq T^{1 / 2} \text { and } s \leq \kappa_{T}
$$

and

$$
p_{s} \leq \frac{2}{T-s} \leq \frac{2 f^{2}(T)}{K T} \leq \frac{2}{K}
$$

if $K \geq 2$.

$$
I \leq \frac{1}{2}\left(1+\frac{2}{K}\right) \sum_{s=1}^{\kappa_{T}} \mathbf{E} \lambda_{s 2}(\mathcal{C}, T-s)
$$

If we assume that $d=1, f(T) \leq T^{1 / 2}$ and $K \geq 2$, then by (3.3), (3.4), (3.5), (3.6) and (3.9), we have

$$
\begin{gathered}
1+(1-\epsilon)\left(1-\frac{1}{K}\right) \frac{c(\alpha)}{2} \frac{T}{f(T)} \leq \mathbf{E}(\lambda(\mathcal{C}, T) \mid \lambda(\mathcal{C}, T)>0) \\
\leq 1+(1+\epsilon) \frac{1}{2}\left(1+\frac{2}{K}\right) c(\alpha) \frac{T}{f(T)}+K \frac{T}{f^{2}(T)} .
\end{gathered}
$$

Hence, we have Theorem 2 in the case $d=1$.

In the case $d=2$, Lemma $3,(3.4)$ and (3.6) imply 


$$
(1-\epsilon)\left(1-\frac{1}{K}\right) \frac{1}{4} \frac{T}{f^{2}(T)} \log f(T) \leq I \leq \frac{1}{2}(1+\epsilon) \frac{T}{f^{2}(T)} \log f(T) .
$$

(3.3), (3.5) and (3.11) imply

$$
\begin{aligned}
& 1+(1-\epsilon)\left(1-\frac{1}{K}\right) \frac{1}{4} \frac{T}{f^{2}(T)} \log f(T) \\
& \leq \mathbf{E}(\lambda(\mathcal{C}, T) \mid \lambda(\mathcal{C}, T)>0) \leq 1+\frac{1}{2}(1+\epsilon) \frac{T}{f^{2}(T)} \log f(T)+K \frac{T}{f^{2}(T)}
\end{aligned}
$$

for any $K>0$.

If we assume that $d=2, f(T) \leq T^{1 / 2}$ and $K \geq 2$, then by (3.3), (3.4), (3.5), (3.6) and (3.9) we have

$$
\begin{gathered}
1+(1-\epsilon)\left(1-\frac{1}{K}\right) \frac{1}{4} \frac{T}{f^{2}(T)} \log f(T) \leq \mathbf{E}(\lambda(\mathcal{C}, T) \mid \lambda(\mathcal{C}, T)>0) \\
\leq 1+\frac{1+\epsilon}{4}\left(1+\frac{2}{K}\right) \frac{T}{f^{2}(T)} \log f(T)+K \frac{T}{f^{2}(T)}
\end{gathered}
$$

Hence, we have Theorem 2 in the case $d=2$.

In the case $d \geq 3$, Lemma $3,(3.4)$ and (3.6) imply

$$
\begin{gathered}
(1-\epsilon)\left(1-\frac{1}{K}\right) \frac{1}{2} \frac{\omega_{d}}{(4 \pi)^{d / 2}} \frac{2}{d-2} \frac{T}{f^{2}(T)} K^{-(d-2) / 2} \\
\leq I \leq(1+\epsilon) \frac{\omega_{d}}{(4 \pi)^{d / 2}} \frac{2}{d-2} \frac{T}{f^{2}(T)} K^{-(d-2) / 2}
\end{gathered}
$$

(3.3), (3.5) and (3.13) imply

$$
\begin{gathered}
1+(1-\epsilon)\left(1-\frac{1}{K}\right) \frac{1}{d-2} \frac{\omega_{d}}{(4 \pi)^{d / 2}} \frac{T}{f^{2}(T)} K^{-(d-2) / 2} \leq \mathbf{E}(\lambda(\mathcal{C}, T) \mid \lambda(\mathcal{C}, T)>0) \\
\leq 1+(1+\epsilon) \frac{\omega_{d}}{(4 \pi)^{d / 2}} \frac{2}{d-2} \frac{T}{f^{2}(T)} K^{-(d-2) / 2}+K \frac{T}{f^{2}(T)}
\end{gathered}
$$

Hence, we have Theorem 2 in the case $d \geq 3$.

Theorem 3 is a simple outcome of the consequences of Theorem 2, Lemma 5 and the following:

Lemma 7:

$$
\mathbf{P}\{\lambda(\mathcal{C}, T)>0 \mid B(T)>0\}=\frac{\mathbf{E}(\lambda(\mathcal{C}, T) \mid B(T)>0)}{\mathbf{E}(\lambda(\mathcal{C}, T) \mid \lambda(\mathcal{C}, T)>0)}
$$

whose proof is trivial.

\section{Proof of Theorem 1}

Let $B(A, T)$ be the number of those particles which are located in $A \subset \mathbb{R}^{d}$ at time $t=0$ and which have at least one offspring living at time $T$. The following lemma is trivial.

Lemma 8:

and

$$
\mathbf{P}\{B(A, T)=k\}=\frac{\nu^{k}}{k !} e^{-\nu} \quad(k=0,1,2, \ldots)
$$

where

as $T \rightarrow \infty$.

$$
\operatorname{Eexp}(-z B(A, T))=\exp \left(\nu\left(e^{-z}-1\right)\right),
$$

$$
\nu=\nu(A, T) \sim \frac{2 \mu|A|}{T},
$$


Introduce the following notations:

$$
\begin{gathered}
\mathrm{C}(R)=\mathrm{C}(0, R), \\
\mathrm{C}_{i}=\mathrm{C}_{i}(\Delta, T)=\mathrm{C}\left(0,(i+1) \Delta T^{1 / 2}\right)-\mathrm{C}\left(0, i \Delta T^{1 / 2}\right), \\
B(R)=B(\mathcal{C}(R), T) \\
B_{i}=B_{i}(\Delta, T)=B\left(\mathcal{C}_{i}, T\right) .
\end{gathered}
$$

Then we have

$$
\begin{aligned}
& |\mathrm{C}(R)|=R^{d} \omega_{d} \\
& =d \omega_{d} \Delta^{d} T^{d / 2} i^{d-1} \leq\left|\mathcal{C}_{i}\right|=\Delta^{d} T^{d / 2}\left((i+1)^{d}-i^{d}\right) \omega_{d} \leq 2^{d} \omega_{d} \Delta^{d} T^{d / 2} i^{d-1}, \\
& \mathbf{E} B(R) \sim \frac{2 \mu \omega_{d} R^{d}}{T} \\
& \exp \left(-(1+\epsilon) \frac{2 \mu \omega_{d} R^{d}}{T}\right) \leq \mathbf{P}\{B(R)=0\} \leq \exp \left(-(1-\epsilon) \frac{2 \mu \omega_{d} R^{d}}{T}\right), \\
& \exp \left(-(1+\epsilon) \frac{2 \mu \omega_{d} R^{d}}{T}\left(e^{-z}-1\right)\right) \leq \operatorname{Eexp}(-z B(R)) \leq \exp \left(-(1-\epsilon) \frac{2 \mu \omega_{d} R^{d}}{T}\left(e^{-z}-1\right)\right) \\
& (1-\epsilon) 2 \mu d \omega_{d} \Delta^{d} i^{d-1} T^{(d-2) / 2} \leq \mathbf{E} B_{i} \sim \frac{2 \mu\left|\mathcal{C}_{i}\right|}{T} \leq(1+\epsilon) 2 \mu 2^{d} \omega_{d} \Delta^{d} i^{d-1} T^{(d-2) / 2}, \\
& \exp \left(-(1+\epsilon) 2 \mu 2^{d} \omega e_{d} \Delta^{d} i^{d-1} T^{(d-2) / 2}\right) \leq \mathbf{P}\left\{B_{i}=0\right\} \\
& \sim \exp \left(-\frac{2 \mu\left|\mathfrak{C}_{i}\right|}{T}\right) \leq \exp \left(-(1-\epsilon) 2 \mu d \omega_{d} \Delta^{d} i^{d-1} T^{(d-2) / 2}\right) \\
& \exp \left((1+\epsilon) 2 \mu 2^{d} \omega_{d} \Delta^{d} i^{d-1} T^{(d-2) / 2}\left(e^{-z}-1\right)\right) \leq \operatorname{Eexp}\left(-z B_{i}\right) \sim \exp \left(\frac{2 \mu\left|\mathrm{C}_{i}\right|}{T}\left(e^{-z}-1\right)\right) \\
& \leq \exp \left((1-\epsilon) 2 \mu d \omega_{d} \Delta^{d} i^{d-1} T^{(d-2) / 2}\left(e^{-z}-1\right)\right) .
\end{aligned}
$$

Now we present the proof of Theorem 1 in eight steps.

Step 1: Let $d=2$ and

Then by (4.2),

$$
R_{1}=R_{1}(T)=K(T \log T)^{1 / 2}
$$

$$
\mathbf{P}\left\{B\left(R_{1}\right)=0\right\} \leq \exp \left(-(1-\epsilon) 2 \mu \pi K^{2} \log T\right) .
$$

Consider a particle which is located in $\mathrm{C}\left(R_{1}\right)$ at time $t=0$ and which has a living offspring at time $T$. Let $V(0)$ be the location of the considered particle at $t=0$ and let $V(T)$ be the location of an arbitrary, fixed offspring of the considered particle at time $T$. Then,

Consequently,

$$
\mathbf{P}\left\{|V(T)-V(0)| \geq R_{1}\right\} \leq \exp \left(-\frac{K^{2}}{2} \log T\right) .
$$

$$
\mathbf{P}\left\{\Lambda\left(\mathcal{C}\left(2 R_{1}\right), T\right)=0\right\} \leq \exp \left(-(1-\epsilon) 2 \mu \pi K^{2} \log T\right)+\exp \left(-\frac{K^{2}}{2} \log T\right) .
$$

Hence, by Borel-Cantelli lemma,

$$
\Lambda\left(\mathcal{C}\left(2 R_{1}\right), T\right)>0, \text { a.s. }
$$

for all but finitely many $T$ provided that

$$
K>\max \left\{2^{1 / 2},(2 \mu \pi)^{-1 / 2}\right\},
$$

Step 2: Let $d=2$ and where

$$
R_{2}=R_{2}(T)=\frac{T^{1 / 2}}{f(T)}
$$




$$
f(T) \uparrow \infty, \quad R_{2}(T) \uparrow \infty
$$

Consider $B_{i}$ particles located in the ring $\mathrm{C}_{i}$ at time $t=0$ having offspring living at time $T$. Let $\lambda_{j}^{(i)}(A, T)\left(j=1,2, \ldots, B_{i}\right)$ be the number of those offspring of the $j$ th particle which are located in $A$ at time $T$. Then, by (1.3),

and by (4.6)

$$
\mathbf{P}\left\{\lambda_{j}^{(i)}\left(\mathcal{C}\left(R_{2}\right), T\right)>0\right\} \leq \frac{\exp \left(-\frac{i^{2} \Delta^{2}}{2}\right)}{\log f(T)}
$$

$$
\begin{gathered}
\mathbf{P}\left\{\prod_{j=1}^{B_{i}}\left\{\lambda_{j}^{(i)}\left(\mathrm{C}\left(R_{2}\right), T\right)=0\right\}\right\}=\mathbf{E P}\left\{\prod_{j=1}^{B_{i}}\left\{\lambda_{j}^{(i)}\left(\mathrm{C}\left(R_{2}\right), T\right)=0\right\} \mid B_{i}\right\} \\
\geq \mathbf{E}\left(-\frac{\exp \left(-\frac{i^{2} \Delta^{2}}{2}\right)}{\log f(T)}\right)^{B_{i}}=\exp \left(\nu\left(e^{-z}-1\right)\right)
\end{gathered}
$$

where

and

$$
\nu=\nu_{i}=\frac{2 \mu\left|\mathrm{C}_{i}\right|}{T} \leq 8 \mu \pi \Delta^{2} i
$$

Hence,

$$
e^{-z}=e^{-z_{i}}=1-\frac{\exp \left(-\frac{i^{2} \Delta^{2}}{2}\right)}{\log f(T)} \text {. }
$$

Since, as $\Delta \rightarrow \infty$,

$$
\begin{aligned}
\mathbf{P}\left\{\Lambda\left(\mathcal{C}\left(R_{2}\right), T\right)=0\right\} & \geq \prod_{i=0}^{\infty} \exp \left(\nu_{i}\left(e^{-z_{i}}-1\right)\right) \geq \exp \left(-\sum_{i=0}^{\infty} 8 \mu \pi i \Delta^{2} \frac{\exp \left(-\frac{i^{2} \Delta^{2}}{2}\right)}{\log f(T)}\right) \\
& \geq \exp \left(-\frac{8 \mu \pi}{\log f(T)} \sum_{i=0}^{\infty} i \Delta^{2} \exp \left(-\frac{i^{2} \Delta^{2}}{2}\right)\right) .
\end{aligned}
$$

we have

$$
\sum_{i=0}^{\infty} i \Delta^{2} \exp \left(-\frac{i^{2} \Delta^{2}}{2}\right) \sim \int_{0}^{\infty} x \exp \left(-\frac{x^{2}}{2}\right) d x=1
$$

$$
\mathbf{P}\left\{\Lambda\left(\mathrm{e}\left(R_{2}\right), T\right)=0\right\} \geq 1-\frac{8 \mu \pi}{\log f(T)} \rightarrow 1 .
$$

Which, in turn, implies

$$
\Lambda\left(\mathcal{C}\left(R_{2}\right), T\right)=0 \quad \text { i.o. } \quad \text { a.s. }
$$

Step 3: Let $d=2$,

Then by Lemma 8 ,

$$
\begin{gathered}
\left.R_{3}=R_{3}(T)=\log T\right)^{-1 / 2+\epsilon}, \quad(\epsilon>0) \\
T_{k}=e^{k}, \quad \rho_{k}=T_{k}^{1 / 2}, \\
R_{3}(k)=R_{3}\left(T_{k}\right) .
\end{gathered}
$$

$$
\begin{gathered}
\mathbf{P}\left\{B\left(\mathcal{C}\left(\rho_{k+1}\right)-\mathcal{C}\left(\rho_{k}\right), T_{k+1}\right)=0\right\} \leq \exp \left(-(1-\epsilon) \frac{2 \mu \pi}{T_{k+1}}\left(\rho_{k+1}^{2}-\rho_{k}^{2}\right)\right) \\
=\exp \left(-(1-\epsilon) \frac{2 \mu \pi(e-1)}{e}\right)<1
\end{gathered}
$$

Consider a particle which is located in $\mathcal{C}\left(\rho_{k+1}\right)-\mathcal{C}\left(\rho_{k}\right)$ at time $t=0$ and which has a living offspring at time $T_{k+1}$. Note that by $(4.12)$ with positive probability there exists such a particle. Let $\lambda_{3}(k)$ be the number of those offspring of the considered particle which are located 
in $\mathrm{C}\left(R_{3}(k+1)\right)$ at time $T_{k+1}$. Then by (1.3),

$$
\mathbf{P}\left\{\lambda_{3}(k)>0\right\} \geq \frac{1-\epsilon}{k+1} e^{-1 / 2} .
$$

Since the events $\left\{\lambda_{3}(k)>0\right\}$ are independent we have

$$
\Lambda\left(\mathbb{C}\left(R_{3}\right), T\right)>0 \quad \text { i.o a.s. }
$$

Step 4: Let $d=2$,

Then by (4.4),

$$
R_{4}=R_{4}(T)=T^{-1 / 2}(\log T)^{-1 / 2-\epsilon} .
$$

$$
\mathbf{E} B_{i} \leq(1+\epsilon) 8 \mu \pi \Delta^{2} i .
$$

Consider the particles located in the ring $\mathrm{C}_{i}$ at time $t=0$ having offspring living at time $T$. Let $\lambda_{j}^{(i)}(A, T)\left(j=1,2, \ldots, B_{i}\right)$ be the number of those offspring of the $j$ th particle which are located in $A$ at time $T$. Then by (1.4),

and

$$
\mathbf{P}\left\{\lambda_{j}^{(i)}\left(\mathcal{C}\left(R_{4}\right), T\right)>0\right\} \leq \frac{R_{4}^{2}}{4} \exp \left(-\frac{i^{2} \Delta^{2}}{2}\right)
$$

Hence,

$$
\begin{gathered}
\mathbf{P}\left\{\sum_{j=1}^{B_{i}} \lambda_{j}^{(i)}\left(\mathrm{e}\left(R_{4}\right), T\right)>0\right\} \leq \mathbf{E P}\left\{\sum_{j=1}^{B_{i}} \lambda_{j}^{(i)}\left(\mathfrak{C}\left(R_{4}\right), R\right)>0 \mid B_{i}\right\} \\
\leq \mathbf{E} B_{i} \frac{R_{4}^{2}}{4} \exp \left(-\frac{i^{2} \Delta^{2}}{2}\right) \leq 2 \mu \pi R_{4}^{2} \Delta^{2} i \exp \left(-\frac{i^{2} \Delta^{2}}{2}\right) .
\end{gathered}
$$

$$
\begin{gathered}
\mathbf{P}\left\{\Lambda\left(\mathcal{C}\left(R_{4}\right), T\right)>0\right\} \leq \sum_{i=0}^{\infty} \mathbf{P}\left\{\sum_{j=1}^{B_{i}} \lambda_{j}^{(i)}\left(\mathcal{C}\left(R_{4}\right), T\right)>0\right\} \\
\leq 2 \mu \pi R_{4}^{2} \sum_{i=0}^{\infty} \Delta^{2} i \exp \left(-\frac{i^{2} \Delta^{2}}{2}\right) .
\end{gathered}
$$

Consequently,

for all but finitely many $T$.

$$
\Lambda\left(\mathcal{C}\left(R_{4}\right), T\right)=0 \quad \text { a.s. }
$$

Step 5: Let $d \geq 3$ and

$$
R_{1}=R_{1}(T)=K(\log T)^{1 /(d-2)} .
$$

Define $\lambda_{j}^{(i)}(\cdot, \cdot)$ as in Step 2. Then by (1.6),

and

$$
\mathbf{P}\left\{\lambda_{j}^{(i)}\left(\mathcal{C}\left(R_{1}\right), T\right)>0\right\} \geq \frac{2^{d} \omega_{d}(d-2)}{8 \omega_{d}+4(8 \pi)^{d / 2}(d-2)} \exp \left(-\frac{(i+1)^{2} \Delta^{2}}{2}\right)\left(\frac{R_{1}}{T^{1 / 2}}\right)^{d-2}
$$

$$
\begin{aligned}
& \mathbf{P}\left\{\prod_{j=1}^{B_{i}}\left\{\lambda_{j}^{(i)}\left(\mathcal{C}\left(R_{1}\right), T\right)=0\right\}\right\}=\mathbf{E P}\left\{\prod_{j=1}^{B_{i}}\left\{\lambda_{j}^{(i)}\left(\mathcal{C}\left(R_{1}\right), T\right)=0\right\} \mid B_{i}\right\} \\
& \leq \mathbf{E}\left(1-M \exp \left(-\frac{(i+1)^{2} \Delta^{2}}{2}\right)\left(\frac{R_{1}}{T^{1 / 2}}\right)^{d-2}\right)^{B_{i}}=\exp \left(\nu\left(e^{-z}-1\right)\right),
\end{aligned}
$$

where

$$
M=\frac{2^{d} \omega_{d}(d-2)}{8 \omega_{d}+4(8 \pi)^{d / 2}(d-2)}
$$




$$
\begin{gathered}
\nu=\nu_{i}=\frac{2 \mu\left|\mathcal{C}_{i}\right|}{T}=\frac{2 \mu \omega_{d}}{T}\left((i+1)^{d}-i^{d}\right) T^{d / 2} \Delta^{d} \geq \frac{2 \mu d \omega_{d}}{T} i^{d-1} T^{d / 2} \Delta^{d}, \\
e^{-z}=e^{-z_{i}}=1-M \exp \left(-\frac{(i+1)^{2} \Delta^{2}}{2}\right)\left(\frac{R_{1}}{T^{1 / 2}}\right)^{d-2} .
\end{gathered}
$$

Hence,

$$
\begin{gathered}
\mathbf{P}\left\{\Lambda\left(\mathcal{C}\left(R_{1}\right), T\right)=0\right\} \leq \prod_{i=0}^{\infty} \exp \left(\nu_{i}\left(e^{-z_{i}}-1\right)\right) \\
\leq \exp \left(-\sum_{i=0}^{\infty} \frac{2 \mu d \omega_{d}}{T} T^{d / 2} i^{d-1} M\left(\frac{R_{L}}{T^{1 / 2}}\right)^{d-2} \Delta^{d} \exp \left(-\frac{(i+1)^{2} \Delta^{2}}{2}\right)\right) \\
\leq \exp \left(-2 \mu d \omega_{d} M K^{d-2} \log T \sum_{i=0}^{\infty} i^{d-1} \Delta^{d} \exp \left(-\frac{(i+1)^{2} \Delta^{2}}{2}\right)\right) \\
2 \mu d \omega_{d} M K^{d-2} \sum_{i=0}^{\infty} i^{d-1} \Delta^{d} \exp \left(-\frac{(i+1)^{2} \Delta^{2}}{2}\right)>1
\end{gathered}
$$

Choose $K$ such that

Then, we have$$
\Lambda\left(\mathcal{C}\left(R_{1}\right), T\right)>0 \text { a.s. }
$$

for all but finitely many $T$.

Step 6: Let $d \geq 3$ and

$$
R_{2}=R_{2}(T)=K(\log \log \log T)^{1 /(d-2)} .
$$

Now follow the proof of Step 2, with the following modifications: instead of (4.8) by (1.6), we have

$$
\mathbf{P}\left\{\lambda_{j}^{(i)}\left(\mathcal{C}\left(R_{2}\right), T\right)>0\right\} \leq 2^{d-2}(d-2) \exp \left(-\frac{i^{2} \Delta^{2}}{2}\right) R_{2}^{d-2} T^{-(d-2) / 2}
$$

instead of (4.9), we have

$$
\nu=\nu_{i}=\frac{2 \mu\left|C_{i}\right|}{T} \leq 2^{d+1} \mu \omega_{d} \Delta^{d} T^{(d-2) / 2} i^{d-1}
$$

instead of (4.10), we have

$$
e^{-z}=e^{-z} i=1-2^{d-2}(d-2) \exp \left(-\frac{i^{2} \Delta^{2}}{2}\right) R_{2}^{d-2} T^{-(d-2) / 2}
$$

instead of (4.11), we have

$$
\mathbf{P}\left\{\Lambda\left(\mathcal{C}\left(R_{2}\right), T\right)=0\right\} \geq \exp \left(-2^{2 d-1} \mu \omega_{d}(d-2) R_{2}^{d-2} \sum_{i=0}^{\infty} i^{d-1} \Delta^{d} \exp \left(-\frac{i^{2} \Delta^{2}}{2}\right)\right) .
$$

Hence, if $K$ is small enough, then

where

$$
\mathbf{P}\left\{\Lambda\left(\mathcal{C}\left(R_{2}\left(T_{k}\right)\right), T_{k}\right)=0\right\} \geq \frac{1}{k}
$$

$$
T_{k}=\exp \left(\exp k^{2}\right) \text {. }
$$

Observe that the probability that at least one particle among the ones who are located in $\mathrm{C}\left(T_{k}\right)$ at time $t=0$ would live at time $T_{k+1}$, is equal to

$$
1-\exp \left(-2 \omega_{d} \mu \frac{T_{k}^{d}}{T_{k+1}}\right) \sim 2 \omega_{d} \mu \frac{T_{k}^{d}}{T_{k+1}}=2 \omega_{d} \mu \exp \left(-e^{k^{2}}\left(e^{2 k+1}-d\right)\right) .
$$

Hence, there is no particle in $\mathrm{C}\left(T_{k}\right)$ living up to time $T_{k+1}$.

Consequently, by Borel-Cantelli lemma we have

$$
\Lambda\left(\mathcal{C}\left(R_{2}\right), T\right)=0 \text { i.o. a.s. }
$$

Step 7: Let $d \geq 3$, 


$$
R_{3}=R_{3}(T)=M(\log T)^{-1 / d}
$$

Now follow the proof of Step 3 with the following modifications: instead of (4.12), we have

$$
\mathbf{P}\left\{B\left(\mathfrak{C}\left(\rho_{k+1}\right)-\mathfrak{C}\left(\rho_{k}\right), T_{k+1}\right)=0\right\} \leq\left(-(1-\epsilon) \frac{2 \mu \omega_{d}}{T_{k+1}}\left(T_{k+1}^{d / 2}-T_{k}^{d / 2}\right)\right) \rightarrow 0 .
$$

Consider the $B_{k}=B\left(\mathbb{C}\left(\rho_{k+1}\right)-\mathfrak{C}\left(\rho_{k}\right), T_{k+1}\right)$ particles located in $\mathrm{C}\left(\rho_{k+1}\right)-\mathrm{C}\left(\rho_{k}\right)$ at time $t=0$ having offspring living at time $T_{k+1}$. Let $\lambda_{j}^{(k)}(A, T)\left(j=1,2, \ldots, B_{k}\right)$ be the number of those offspring of the $j$ th particle which are located in $A$ at time $T$. Then by (1.7) (with $\beta=0$ ), we have

and

$$
\begin{gathered}
\mathbf{P}\left\{\lambda_{j}^{(k)}\left(\mathcal{C}\left(R_{3}\right), T_{k+1}\right)>0\right\} \geq(1-\epsilon) \frac{\omega_{d} e^{-1 / 2}}{2(2 \pi)^{d / 2} M^{d}} \frac{T_{k+1}}{\left(T_{k+1}\right)^{d / 2} \log T_{k+1}} \\
=(1-\epsilon) \frac{\omega_{d} e^{-1 / 2}}{2(2 \pi)^{d / 2} M^{d}} \frac{R_{3}^{d}\left(T_{k+1}\right)}{T_{k+1}^{(d-2) / 2}}
\end{gathered}
$$

$$
\begin{gathered}
\mathbf{P}\left\{\Lambda\left(\mathcal{C}\left(R_{3}\left(T_{k+1}\right)\right), T_{k+1}\right)=0\right\}=\mathbf{E P}\left\{\Lambda\left(\mathcal{C}\left(R_{3}\left(T_{k+1}\right)\right), T_{k+1}\right)=0 \mid B_{k}\right\} \\
\leq \mathbf{E} \exp \left(-z B_{k}\right)=\exp \left(\nu\left(e^{-z}-1\right)\right),
\end{gathered}
$$

where

and

$$
\nu=\nu_{k}=\frac{2 \mu}{T_{k+1}} \omega_{d}\left(\rho_{k+1}^{d}-\rho_{k}^{d}\right)
$$

Hence,

$$
e^{-z}=e^{-z} k=1-(1-\epsilon) \frac{\omega_{d} e^{-1 / 2}}{2(2 \pi)^{d / 2} M^{d}} \frac{R_{3}^{d}\left(T_{k+1}\right)}{T_{k+1}^{(d-2) / 2}}
$$

$$
\begin{gathered}
\mathbf{P}\left\{\Lambda\left(\mathcal{C}\left(R_{3}\left(T_{k+1}\right)\right), T_{k+1}\right)>0\right\} \\
\geq 1-\exp \left(-\frac{2 \mu}{T_{k+1}} \omega_{d}\left(\rho_{k+1}^{d}-\rho_{k}^{d}\right)(1-\epsilon) \frac{\omega_{d} e^{-1 / 2}}{2(2 \pi)^{d / 2} M^{d}} \frac{R_{3}^{d}\left(T_{k+1}\right)}{T_{k+1}^{(d-2) / 2}}\right) \geq \frac{1}{k}
\end{gathered}
$$

if $M$ is small enough. Consequently,

$$
\Lambda\left(\mathrm{C}\left(R_{3}(T)\right), T\right)>0 \text { i.o. a.s. }
$$

Step 8: Let $d \geq 3$ and

$$
R_{4}=R_{4}(T)=T^{-1 / d}(\log T)^{-1 / d-\epsilon} .
$$

Now follow the proof of Step 4 with the following modifications: instead of (4.14) we have

$$
\mathbf{E} B_{i} \leq 2^{d+1} \mu \omega_{d} \Delta^{d} i^{d-1} T^{(d-2) / 2}
$$

instead of (4.15), by (1.7) we have

instead of (4.16), we have

$$
\mathbf{P}\left\{\lambda_{j}^{(i)}\left(\mathcal{C}\left(R_{4}\right), T\right)>0\right\} \leq \frac{\omega_{d} \exp \left(-\frac{i^{2} \Delta^{2}}{2}\right)}{2(2 \pi)^{d / 2}} R_{4}^{d} T^{-(d-2) / 2}
$$

$$
\mathbf{P}\left\{\sum_{j=1}^{B_{i}} \lambda_{j}^{(i)}\left(\mathcal{C}\left(R_{4}\right), T\right)>0\right\} \leq \mu \omega_{d}^{2}\left(\frac{2}{\pi}\right)^{d / 2} i^{d-1} \Delta^{d} \exp \left(-\frac{i^{2} \Delta^{2}}{2}\right) R_{4}^{d}
$$


instead of (4.17), we have

$$
\mathbf{P}\left\{\Lambda\left(\mathcal{C}\left(R_{4}\right), T\right)>0\right\} \leq \mu \omega_{d}^{2}\left(\frac{2}{\pi}\right)^{d / 2} R_{4}^{d} \sum_{i=0}^{\infty} i^{d-1} \Delta^{d} \exp \left(-\frac{i^{2} \Delta^{2}}{2}\right) .
$$

Consequently,

$$
\Lambda\left(\mathbb{C}\left(R_{4}\right), T\right)=0 \quad \text { a.s. }
$$

for all but finitely many $T$.

\section{References}

[1] Révész, P., Random Walks of Infinitely Many Particles, World Scientific, Singapore 1994. 


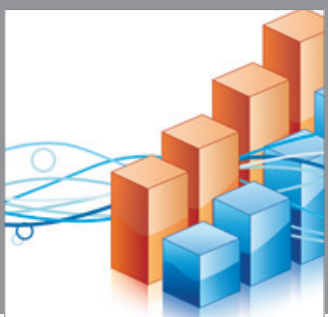

Advances in

Operations Research

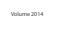

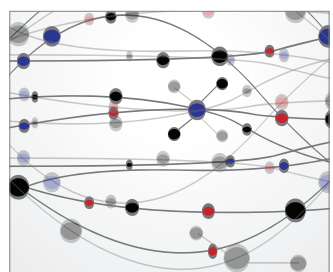

\section{The Scientific} World Journal
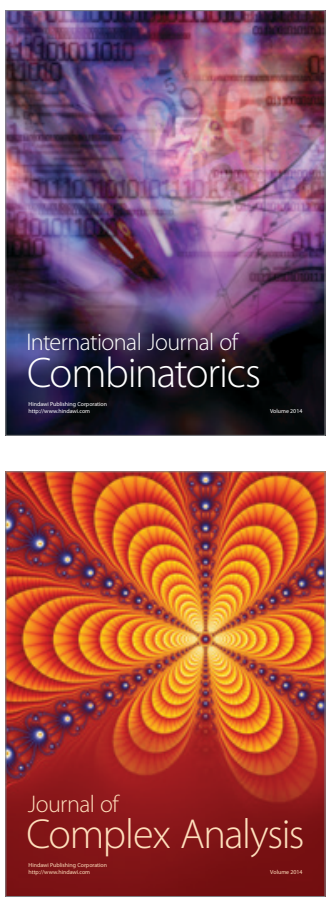

International Journal of

Mathematics and

Mathematical

Sciences
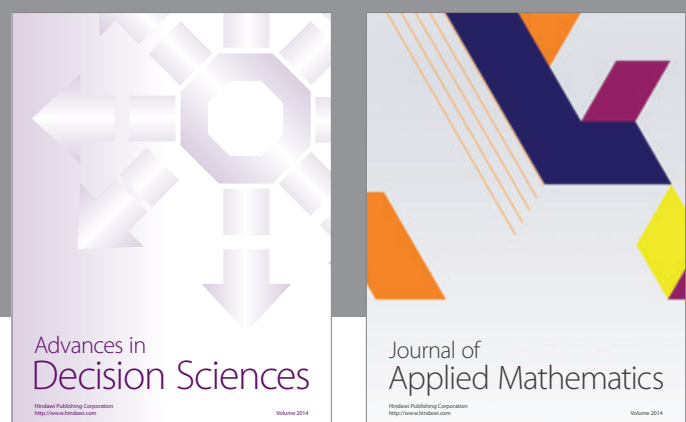

Journal of

Applied Mathematics
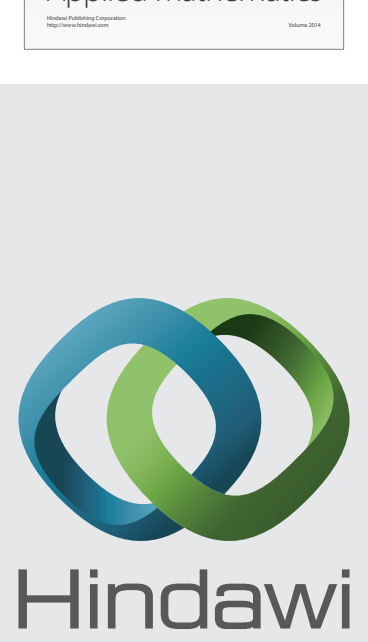

Submit your manuscripts at http://www.hindawi.com
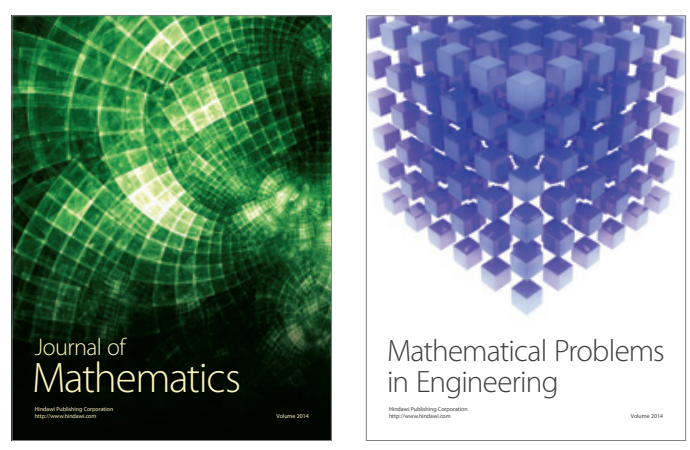

Mathematical Problems in Engineering
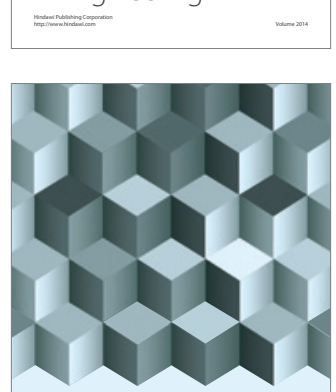

Journal of

Function Spaces
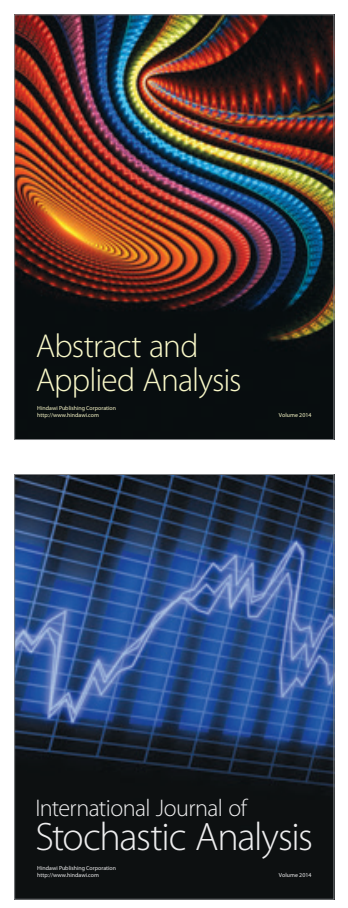

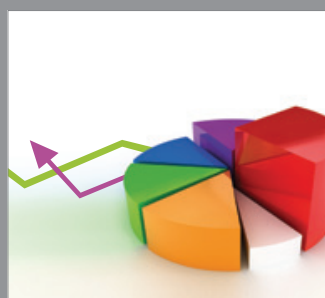

ournal of

Probability and Statistics

Promensencen
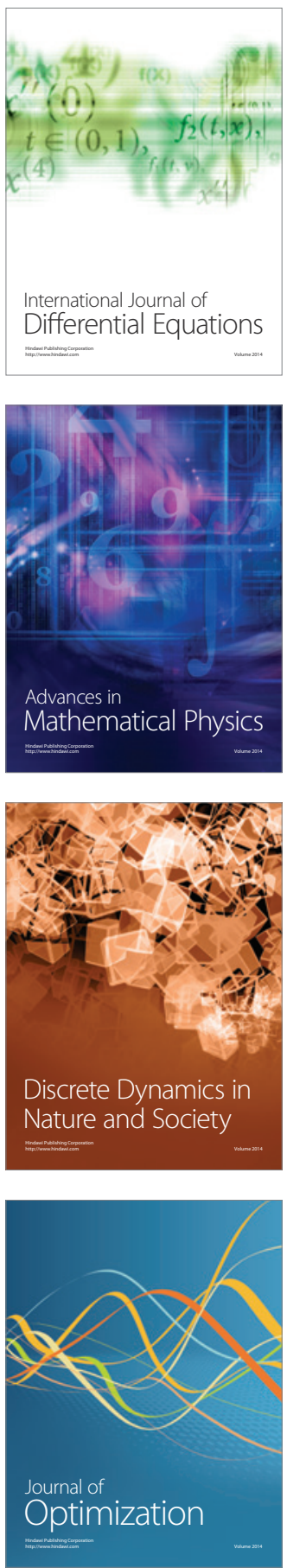